\title{
Promoting parent-child relationships and preventing violence via home-visiting: a pre-post cluster randomised trial among Rwandan families linked to social protection programmes
}

Theresa S. Betancourt ${ }^{*}$, Sarah K. G. Jensen ${ }^{1}$, Dale A. Barnhart ${ }^{2}$, Robert T. Brennan ${ }^{1,3}$, Shauna M. Murray ${ }^{1}$, Aisha K. Yousafzai ${ }^{4}$, Jordan Farrar ${ }^{1}$, Kalisa Godfroid ${ }^{5}$, Stephanie M. Bazubagira ${ }^{5}$, Laura B. Rawlings ${ }^{6}$, Briana Wilson ${ }^{6}$, Vincent Sezibera ${ }^{7}$ and Alex Kamurase ${ }^{6}$

\begin{abstract}
Background: Sugira Muryango is a father-engaged early child development and violence-prevention home-visiting programme delivered by trained lay workers. This cluster-randomised trial evaluates whether families living in extreme poverty (Ubudehe 1, the poorest category in the Government of Rwanda's wealth ranking) who receive Sugira Muryango in combination with a government-provided social protection programme demonstrate greater responsive, positive caregiving, nutrition, care seeking, hygiene, and father involvement compared with control families receiving usual care (UC).

Methods: Using detailed maps, we grouped closely spaced villages into 284 geographic clusters stratified by the type of social protection programmes operating in the village clusters; 198 clusters met all enrolment criteria. Sugira Muryango was delivered to $n=541$ families in 100 treatment clusters with children aged 6-36 months living in extreme poverty. We assessed changes in outcomes in intervention and $n=508 \mathrm{UC}$ control families using structured surveys and observation. Analyses were intent to treat using mixed models to accommodate clustering. (Continued on next page)
\end{abstract}

\footnotetext{
* Correspondence: theresa.betancourt@bc.edu

'Boston College School of Social Work, McGuinn Hall 106M, 140 Commonwealth Avenue, Chestnut Hill, MA 02467, USA

Full list of author information is available at the end of the article
}

(c) The Author(s). 2020 Open Access This article is licensed under a Creative Commons Attribution 4.0 International License, which permits use, sharing, adaptation, distribution and reproduction in any medium or format, as long as you give appropriate credit to the original author(s) and the source, provide a link to the Creative Commons licence, and indicate if changes were made. The images or other third party material in this article are included in the article's Creative Commons licence, unless indicated otherwise in a credit line to the material. If material is not included in the article's Creative Commons licence and your intended use is not permitted by statutory regulation or exceeds the permitted use, you will need to obtain permission directly from the copyright holder. To view a copy of this licence, visit http://creativecommons.org/licenses/by/4.0/ The Creative Commons Public Domain Dedication waiver (http://creativecommons.org/publicdomain/zero/1.0/) applies to the data made available in this article, unless otherwise stated in a credit line to the data. 


\begin{abstract}
(Continued from previous page)
Results: Families receiving Sugira Muryango improved on core outcomes of parent-child relationships assessed using the Home Observation for Measurement of the Environment (Cohen's $d=0.87,95 \% \mathrm{Cl}: 0.74,0.99)$ and the Observation of Mother-Child Interaction (Cohen's $d=0.29,95 \% \mathrm{Cl}: 0.17,0.41$ ). We also saw reductions in harsh discipline on items from the UNICEF MICS (OR $=0.30: 95 \% \mathrm{Cl}: 0.19,0.47)$ and in violent victimisation of female caregivers by their partners $(\mathrm{OR}=0.49,95 \% \mathrm{Cl}: 0.24,1.00)$ compared with UC. Moreover, children in families receiving SM had a 0.45 higher increase in food groups consumed in the past $24 \mathrm{~h}$ (Cohen's $d=0.35,95 \%$ Cl: 0.22 , 0.47), increased care seeking for diarrhoea ( $\mathrm{OR}=4.43,95 \% \mathrm{Cl}: 1.95,10.10)$ and fever $(\mathrm{OR}=3.28,95 \% \mathrm{Cl}: 1.82,5.89)$, and improved hygiene behaviours such as proper treatment of water $(\mathrm{OR}=3.39,95 \% \mathrm{Cl}: 2.16,5.30)$ compared with UC. Finally, Sugira Muryango was associated with decreased caregiver depression and anxiety $(\mathrm{OR}=0.58,95 \% \mathrm{Cl}$ : $0.38,0.88)$.
\end{abstract}

Conclusions: Sugira Muryango led to improvements in caregiver behaviours linked to child development and health as well as reductions in violence.

Trial registration: ClinicalTrials.gov number NCT02510313.

Keywords: Home-visiting, Early childhood development (ECD), Violence, Social protection, Father engagement, Poverty

\section{Background}

Children living in poverty face multiple risks to healthy development including malnutrition, illness, understimulating environments, and harsh discipline [1]. Addressing these adversities is critical during early childhood when ongoing neural development makes the brain particularly plastic to environmental influences and the rapid achievement of developmental milestones gives rise to cognitive and emotional changes occurring at a speed unparalleled in any other developmental period [2]. Social protection programmes target poor households where early child development (ECD) deficits are concentrated, such as in poor rural settings, where income support can increase investment in dietary diversity, hygiene, and responsive caregiving [3]. Previous ECD interventions conducted in low- and middleincome countries (LMICs) demonstrate the value of parenting interventions in improving children's health and development $[4,5]$. Moreover, integrated interventions that build parenting content into other interventions such as social protection and nutrition programmes may offer opportunities for synergistic effects on the home environment and parent-child relationships [6]. For example, engagement and education of caregivers can further improve child health and development through behavioural change [3], and engagement of male caregivers in parent-child interaction and caregiving can help reduce family violence [7].

Rwanda is a low-income country in Sub-Saharan Africa. Although poverty rates in Rwanda have declined in recent years, 38\% of Rwandans still live in poverty (defined as yearly consumption per adult equal to or less than RWF 159,375/US\$207 in 2016), and 16\% live in extreme poverty (defined as yearly consumption per adult of equal to or less than RWF 105,064/US\$136 in 2016)
[8]. These definitions which equal living on less than US $\$ 0.60$ or US $\$ 0.40$ per day, respectively, are well below the World Bank definition of extreme poverty as living on less than US $\$ 1.90$ per day [9]. The Government of Rwanda is addressing poverty-related disparities in ECD via their Vision 2020 Umurenge Program (VUP), which targets nutrition and ECD among the poorest households, offering direct support (unconditional cash transfers), nutrition-sensitive direct support, and public works programming [10]. Rwanda has a robust ECD policy that has been explicitly linked to its Economic Development and Poverty Reduction Strategy [11]. In this manner, the VUP poverty reduction programme provides a platform for targeting the most vulnerable households to promote ECD and prevent violence given high rates of difficulties in both of these areas among families in extreme poverty [12].

In this paper, we evaluate the Sugira Muryango (Strengthen the Family) home-visiting ECD coaching programme delivered in combination with Rwanda's VUP support by community-based coaches (CBCs; see Table 1 for selection criteria, training, supervision, and incentives of $\mathrm{CBCs}$ ). We report data from a cluster randomised trial of 1049 families living in extreme poverty. Sugira Muryango comprises five core components (see Theory of Change, Fig. 1): 1) providing psychoeducation on children's development, nutrition, health, and hygiene promotion; 2) coaching caregivers in active stimulation (play and communication) and responsive parenting to promote "serve-and-return" interactions; 3) reducing family violence via father engagement and improved conflict resolution and parental emotion regulation skills; 4) strengthening problem-solving skills and social support through access to available informal and formal resources; and 5) building skills in positive parenting 
Table 1 Community Based Coaches (CBCs): training, supervision and incentives

- Selection criteria: a) Live in the beneficiary households they will deliver the intervention to; b) be Rwandan; c) Be aged 18 or older; d) be able to write, read, and count in Kinyarwanda, d) be committed to young children and family values e) have the required amount of time to carry out the Sugira Muryango intervention with a select number of households; d) be recommended and approved by local community and authorities

- Three-week training session delivered by trained supervisors.

- Training included role-play-based learning, active coaching practice, techniques for engaging fathers, strategies for providing feedback to caregivers on early stimulation, conflict resolution, problem solving, and resource navigation.

- Supervision provided by Sugira Muryango supervisors who had been involved in previous pilot work.

- Supervision took the form of in-person supervision of the CBCs during the first three weeks of the intervention, and each supervisor shadowed each CBC once in the home. Telephone supervision and peer support groups occurred weekly, and group supervision was held once a month.

- CBCs audiotaped the home-based sessions, which were reviewed by a supervisor for fidelity monitoring.

CBCs were stipended according to local practices (28,000 Rwandan Francs per month for a caseload of five families), visiting weekly for a period of three months and participating in all training and supervision

and coping skills to promote healthy family functioning. These components are tailored towards observed challenges in nurturing care as identified in a report on "Knowledge, Attitudes and Practices Assessment on Early Nurturing of Children" [12]. This report found that family violence including harsh discipline and intimate partner violence were common threats to healthy child development in Rwanda, particularly among poor caregivers who more commonly reported engaging in harsh discipline, including slapping and shouting at the child, compared with families in the higher socioeconomic categories. Moreover, the same report highlighted that fathers in Rwanda are traditionally viewed as the providers for the household rather than active participants in childcare. Key features of emphasis for Sugira Muryango are therefore the program's attention to violence reduction and increasing father engagement in play and caregiving.

\section{Methods}

\section{Study design}

Between January and September 2018, we conducted baseline and post-intervention assessments of a stratified cluster-randomised trial designed to test Sugira Muryango's effects on promoting ECD and preventing violence among families receiving VUP. The trial was conducted within the Nyanza, Ngoma, and Rubavu districts with existing VUP programmes, selected to minimise the overlap with ECD interventions by government or nongovernmental organisations. All families were eligible for one of two versions of the VUP programme: classic public works (cPW), which provides cash for (typically hard) manual labour; or the newer expanded public works (ePW), which provides cash for (typically lighter) labour and also provides access to livestock. All procedures were approved by the Harvard T. H. Chan School of Public Health and Boston College Institutional Review Boards as well as the Rwanda National Ethics Committee, National Committee

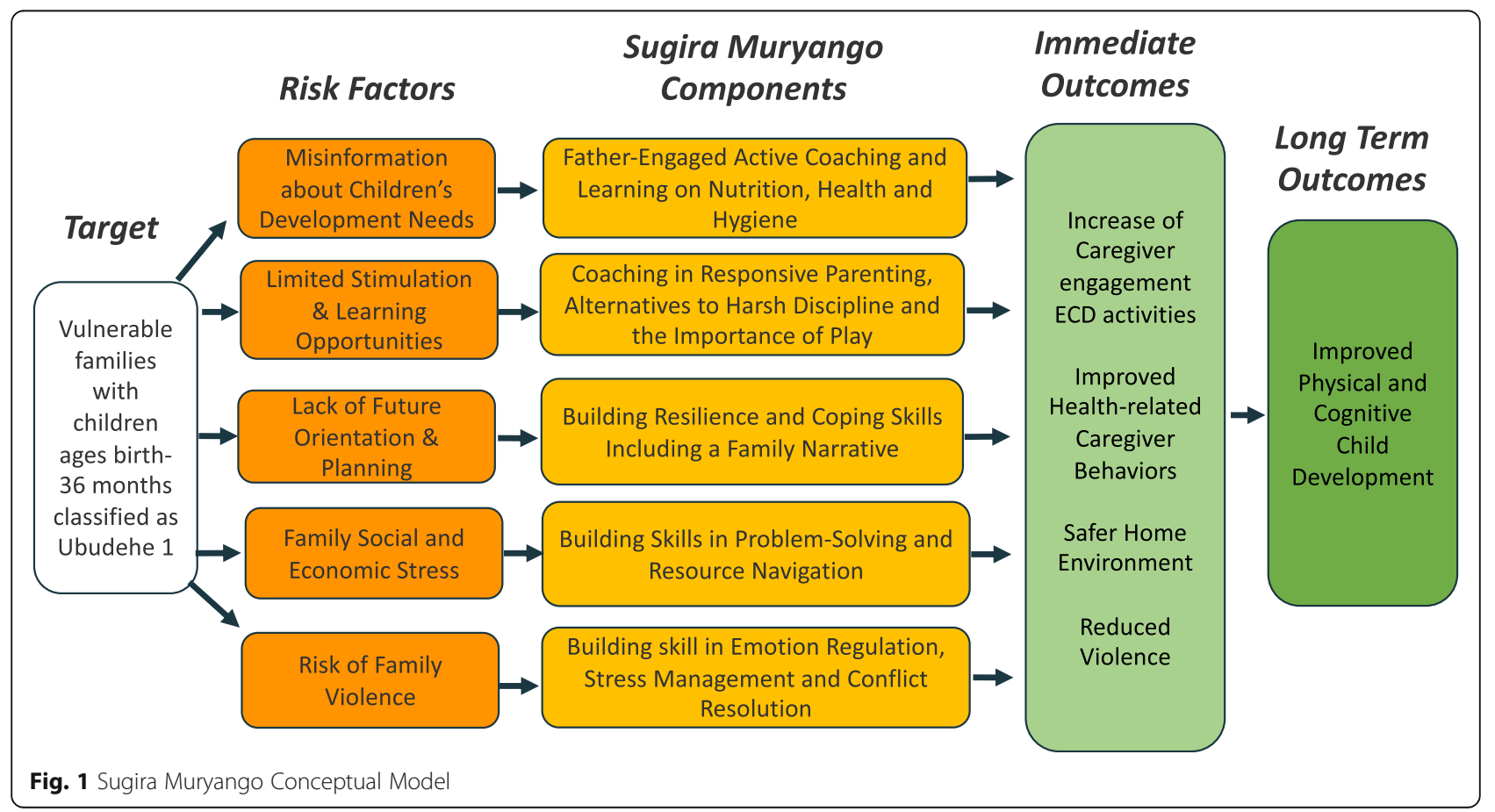


for Science and Technology, and the National Institute of Statistics of Rwanda.

\section{Participants}

Within selected clusters (described in Randomisation, below), families were eligible for inclusion in the study if they 1) belonged to the most extreme level of poverty in the government's household-ranking system (Ubudehe 1) [13] and were eligible for the $\mathrm{cPW}$ or ePW programme; 2) had at least one child 6-36 months; and 3) were willing to participate in a home-visiting intervention. The focus of the programme on children aged 6-36 months at enrolment was based on the knowledge that experiences, both social and biological, are particularly important during the first few years of development [3]. Furthermore, Sugira Muryango is designed to aid child development to the point where government child care becomes available at age 5; at 12-month follow up in the present study the oldest children will be reaching roughly 4.5 years old. Exclusion criteria were a severe, active crisis in the family such as psychosis or suicide attempts by a caregiver or severe mental impairment in the caregiver, which may have affected the ability to benefit from the programme. All caregivers gave written informed consent for themselves and their eligible children.

The caregiver who stated that he or she knew the child best-typically the mother-provided reports on child development and health, the home environment, caregiverchild relationships, caregiving practices, feeding practices, child health, as well as information about the household, including family composition and assets. All primary male and female caregivers provided self-reports on mental health and victimisation and perpetration of intimate partner violence. Interviews, child assessments, and motherchild observation were conducted in Kinyarwanda in the family's home. Data were entered on Android tablets by independent local enumerators blinded to intervention status.

\section{The intervention}

Sugira Muryango comprises 12 modules (see Table 2) that were delivered by trained, supervised CBCs in the families' homes, unless contraindicated due to illness or privacy concerns, at a pace of about one module per week (average 90-min sessions) between May and August 2018. Sugira Muryango offers active coaching of caregivers to promote early stimulation, play, nutrition, hygiene, responsive parenting, nonviolent interactions among household members, and engagement of both female and male caregivers. $\mathrm{CBCs}$ also help families navigate formal and nonformal resources (e.g. health and nutrition services and social support). Sugira Muryango was originally developed and tested in Rwanda for families affected by HIV/ AIDS [14]. During previous pilot studies [15], a version
Table 2 The twelve Sugira Muryango modules

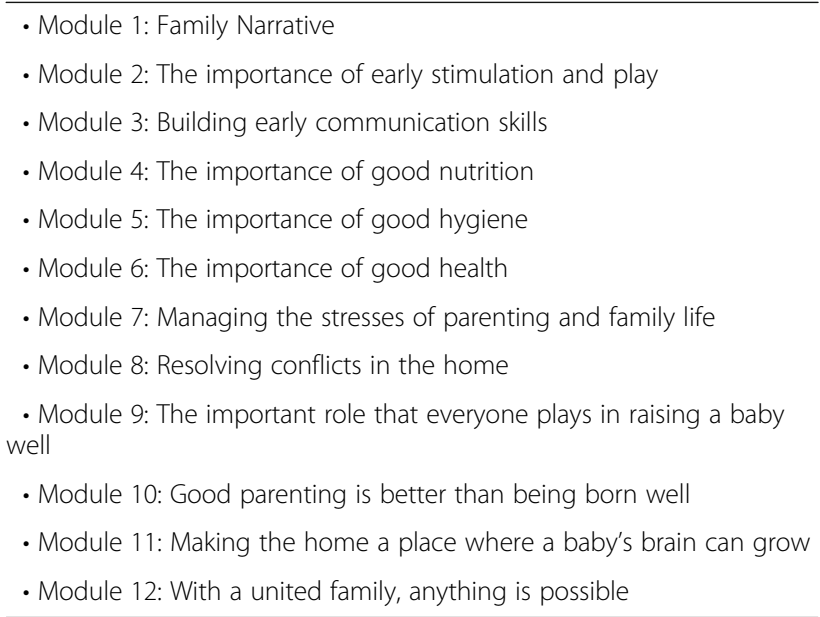

focusing on ECD was developed by integrating United Nations International Children's Emergency Fund (UNICEF) and World Health Organization (WHO) Care for Child Development materials [16]. The CBCs were selected from the local community (see Table 1 for selection criteria, training, supervision, and incentives of CBCs). Primary caregivers participated in the modules in interaction with their child (ren); other caregivers and older children were invited to participate. All visits included a 15-min "active play and communication" session where caregivers received live feedback on parent-child interactions. The usual care (UC) group received VUP services and health services as usual from the Rwandan government and its partners. Intervention and UC families received a stipend (RWF 5000 equivalent to $3 \mathrm{k}$ of rice) after each data collection.

\section{Outcomes}

Per our theory of change (Fig. 1), immediately following 12 modules of intervention delivered weekly over a 3-4 month period, the primary outcomes were change in parents' behaviours towards the child including responsive care and play, dietary diversity, care seeking for child health problems, and family violence. Secondary outcomes were caregiver outcomes related to shared decision-making among parents and caregiver mental health, as well as household outcomes related to water, sanitation, and hygiene. Questionnaires were developed and tested during pilot intervention research and followed a forward- and back-translation protocol from English to Kinyarwanda [14].

With regard to child-level outcomes, responsive caregiving was assessed by trained enumerators using three tools, the Observation of Mother-Child Interaction (OMCI) [17], an adapted 43-item version of the infant/ toddler Home Observation for Measurement of the 
Environment (HOME) Inventory, which has previously been used in Uganda [18, 19], and the Multiple Indicator Cluster Survey (MICS) Family Care Indicators (FCI) [20]. The OMCI assesses a five-minute mother-child interaction that is scored according to published guidelines (maximum 57; Cronbach's $\alpha=0.91$ ). The HOME combines observation of parenting behaviours and household conditions with caregiver report. Items were summed to derive a total score (maximum 43; Cronbach's $\alpha=0.76$ ). The MICS FCI ${ }^{20}$ is a cumulative score of caregivers' self-reported engagement in stimulating activities such as singing, reading, and counting with the child during the prior 3 days (maximum 6; Cronbach's $\alpha=0.74$ ). Children's nutritional intake was assessed by parent-reported dietary diversity reflecting the number of seven food groups (grains, roots, and tubers; legumes and nuts; dairy products; meat, fish, poultry, and organ meats; eggs; vitamin A rich fruits and vegetables; other fruits and vegetables) the child had consumed in the past $24 \mathrm{~h}$ [21]. Children's health status was measured using standard Demographic and Health Surveys (DHS) questions reporting the prevalence of diarrhoea, fever, and cough in the 7 days preceding the survey [22]. Care seeking at a health facility was defined following DHS guidelines and was assessed only among parents of children who experienced illness.

Caregiver-level outcomes included intimate partner violence which was assessed among parents who were currently married, cohabitating, or in a relationship using items from DHS Domestic Violence Module [23] covering emotional, physical, and sexual abuse within the last 3 months. Among households with a motherfather structure, we also assessed whether caregivers reported equal involvement in decision-making about care for the young child including decisions related to feeding and medical care for illness [24]. Finally, caregivers' mental health was assessed using the Hopkins Symptom Checklist-25 (HSCL-25), a measure of depression and anxiety (internalising) symptoms validated for use among adults in Rwanda [25] $(\alpha=0.93)$. A mean score $\geq$ 1.75 was used to define likely clinical mental health problem.

Household-level outcomes related to hygiene practices were assessed using items from the DHS water, sanitation, and hygiene (WASH) module [22]. Indicators included access to clean water, safe treatment of water, and hand-washing facility with soap.

\section{Power calculation}

Power calculations drew on previously conducted pilot studies [15] and estimated the required sample size for a 0.18 minimum detectable standardised effect size on parenting outcomes and child development outcomes for the 3- and 12-month follow-up period assuming power of 0.8 and a standard alpha level of $p<0.05$. We used an estimated intraclass correlation of 0.03 for parent-child interactions based on pilot data. The ePW programme was being rolled out during the design phase of the programme, and we assumed based on estimates available to us it would be too scarce to constitute one half of an ideal sample size, so calculations were based on an assumption of $91 \mathrm{ePW}$ clusters and $104 \mathrm{cPW}$ clusters with five households per cluster to be assigned to treatment and control conditions. Because the target number of all $\mathrm{ePW}$ clusters did not exist, further adjustments were made, by adding combined clusters, and ultimately adding more $\mathrm{cPW}$ clusters to maintain power.

\section{Randomisation}

Families were enrolled between February and March 2018. Government staff in the three study districts provided lists of households participating in $\mathrm{cPW}$ or ePW. Families' participation in VUP was determined by governmental policies and was not under the control of the researchers. Non-overlapping, geographically defined clusters were created comprising at least 30 families participating in the $\mathrm{CPW}$ programme or 10 families participating in the ePW programme, with some clusters containing both $\geq 30 \mathrm{cPW}$ and $\geq 10 \mathrm{ePW}$ households. Clusters were formed by the research team using detailed local maps by combining one or more contiguous villages such that one $\mathrm{CBC}$ could provide services to all participating families in the cluster. Villages within the same cluster were selected to be as close to each other and as far apart from other clusters as possible. Due to the relative scarcity of the ePW families, $100 \%$ of clusters containing at least $10 \mathrm{ePW}$ families were sampled for participation in the study. Clusters which contained cPW families (including combined clusters containing ePW families) were randomly sampled for inclusion into our study until we reached our target sample size of $\geq 1040$ households. Randomisation was performed by a data collection contractor and occurred at the cluster level within strata defined by public works type (ePW only, combined ePW/cPW, and cPW only) and geographic sector. Within strata, clusters were assigned random numbers and placed on a ranked list. The first half of clusters on the randomly ranked list were assigned to treatment. In case of an odd number of clusters per strata, a lottery was used to round the number assigned to treatment up or down. After assignment of the cluster, households were contacted by the data collection contractor and invited to participate in the study. Clusters were retained if at least five families in the $\mathrm{cPW}$ strata or at least one eligible family in the ePW strata enrolled. We retained $48 \mathrm{ePW}$-only clusters, $38 \mathrm{ePW} /$ cPW clusters, and $112 \mathrm{cPW}$-only clusters (Cluster sampling strategy, Fig. 2). Neither the caregivers nor 
enumerators knew the family's assignment status at the time of the baseline assessments. Enumerators were also not informed about the family's assignment status during the post-intervention assessment although caregivers' responses to fidelity questions about the programme following the assessment may have revealed their treatment status. In total, 1049 households were enrolled at baseline. After the randomisation $n=508$ families were allocated to $\mathrm{UC}$ and $n=541$ families were allocated to treatment. Baseline data collection occurred in May 2018 and post-intervention data were collected in August-September 2018.

\section{Statistical analysis}

We compared trajectories of outcomes over time among families receiving the Sugira Muryango intervention with UC using linear mixed models for continuous outcomes and generalised linear mixed models with a logit link for binary outcomes. To account for clustering, we included random effects for randomisation cluster and child for outcomes assessed at the child level. For outcomes assessed at the caregiver level we included random effects for cluster and caregiver, and for outcomes assessed at the household level we included random effects for cluster and household. Following intention-totreat analysis, we used chained equation imputations in STATA to account for missing data [26]. We report coefficients for the time-by-treatment interaction term and standardised effect sizes estimated based on the modelling results (Cohen's $d$ for continuous outcomes, odds ratios (OR) for dichotomous outcomes) with 95\% confidence intervals. Analyses were conducted using StataSE version 15 (StataCorp, College Station, TX). Intraclass correlations can be found in Additional File 1, and further analyses examining whether a family's enrolment in either $\mathrm{ePW}$ or $\mathrm{cPW}$ moderated intervention effects can be found in Additional File 2.

\section{Adverse events in intervention and control households}

During the interval between baseline and postintervention "risk of harm" cases were reported in 12 families $(2.2 \%)$ in the intervention group and 12 families (2.4\%) in the control group (see Additional File 3 for more information). These households were retained in the analyses under intention to treat.

\section{Analytic sample and demographics of the samples}

Baseline data were collected on 1084 children and 1498 caregivers who were enrolled in the trial at baseline. Instances of loss-to-follow-up from baseline to the postintervention assessment was low $(<2.5 \%)$. More specifically, three households $(0.3 \%), 36$ caregivers $(2.4 \%)$, and six children $(0.5 \%)$ did not complete the post-intervention assessment and had post-intervention data imputed. Item-level

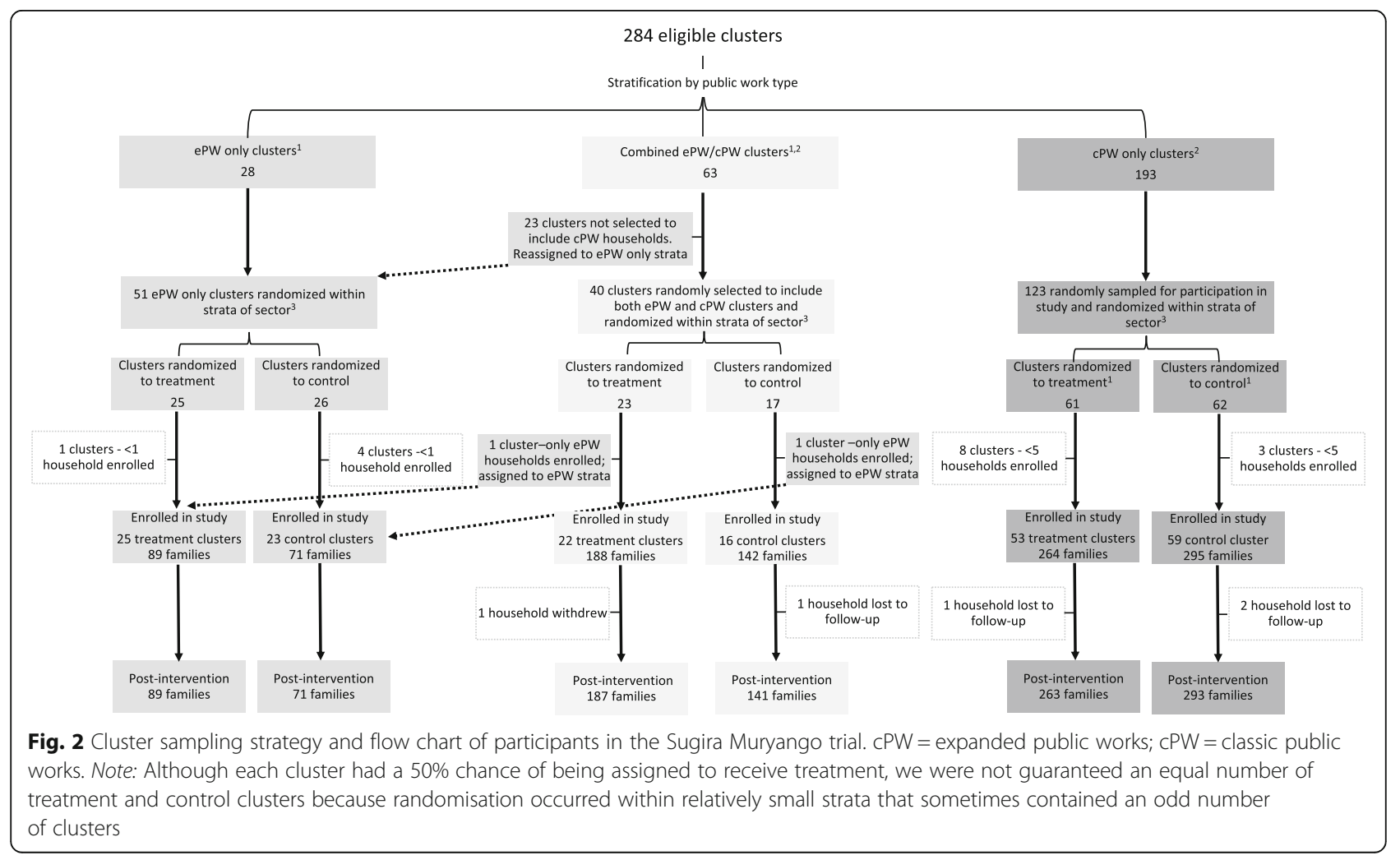


missing data at both baseline and post-intervention were similarly low $(<1 \%)$. Descriptive statistics are provided in Table 3. Caregivers ranged in age from 18 to 79 years and were most frequently the biological mother $(n=950)$, the biological father $(n=433)$, or a grandparent $(n=96)$. Sixty-four percent $(n=953)$ of the caregivers were married or cohabitating. At enrolment, $61 \%$ of the families reported high levels of food insecurity and $48 \%$ of the children were stunted as defined by a standardised heightfor-age (HAZ) score at or below -2SD of the reference population in accordance with WHO growth standards [27].

\section{Results}

Results from the mixed models are shown in Table 4 . Unadjusted baseline and post-intervention means for

Table 3 Descriptive statistics of study participants at enrolment. Continuous variables reported as [mean (SD)] and binary variables reported as [frequency (\%)]

\begin{tabular}{|c|c|c|c|c|}
\hline & \multicolumn{2}{|c|}{ CLASSIC PUBLIC WORKS (CPW) } & \multicolumn{2}{|c|}{ EXPANDED PUBLIC WORKS (ePW) } \\
\hline & Sugira Muryango + CPW & CPW only & Sugira Muryango $+\mathrm{ePW}$ & ePW only \\
\hline HOUSEHOLDS $(N=1049)$ & $n=374$ & $\mathrm{n}=374$ & $n=167$ & $n=134$ \\
\hline High food insecurity & $239(63.9 \%)$ & $229(61.2 \%)$ & $104(62.3 \%)$ & $70(52.2 \%)$ \\
\hline CHILDREN $(N=1084)$ & $n=386$ & $n=384$ & $n=173$ & $n=141$ \\
\hline Average age in months & $21.0(8.14)$ & $21.8(8.6)$ & $20.8(8.2)$ & $22.3(8.4)$ \\
\hline \multicolumn{5}{|l|}{ Health status and wellbeing } \\
\hline Stunted $(\mathrm{HAZ}<2)$ & $184(47.7 \%)$ & $178(46.4 \%)$ & $85(49.1 \%)$ & $75(53.2 \%)$ \\
\hline Wasted $(\mathrm{WHZ}<2)$ & $13(3.4 \%)$ & $9(2.3 \%)$ & $8(4.6 \%)$ & $2(1.4 \%)$ \\
\hline Underweight (WAZ <2) & $63(16.3 \%)$ & $71(18.5 \%)$ & $30(17.3 \%)$ & $27(19.1 \%)$ \\
\hline Screens positive, disability or developmental delay & $110(28.6 \%)$ & $111(29.0 \%)$ & $57(32.9 \%)$ & $38(27.1 \%)$ \\
\hline Any violent punishment & $184(47.7 \%)$ & $180(47.0 \%)$ & $83(48.0 \%)$ & $59(41.8 \%)$ \\
\hline CAREGIVERS $(N=1498)$ & $n=555$ & $n=564$ & $n=211$ & $n=168$ \\
\hline $\begin{array}{l}\text { Average age in years } \\
\text { [range] }\end{array}$ & $\begin{array}{l}34.5(9.7) \\
{[18-79]}\end{array}$ & $\begin{array}{l}35.7(10.3) \\
{[19-75]}\end{array}$ & $\begin{array}{l}36.3(10.6) \\
{[18-79]}\end{array}$ & $\begin{array}{l}37.5(12.7) \\
{[18-84]}\end{array}$ \\
\hline \multicolumn{5}{|l|}{ Marital Status } \\
\hline Single, separated, divorced, widowed & $171(30.8 \%)$ & $166(29.43 \%)$ & 117 (55.5\%) & $91(54 \%)$ \\
\hline Married/cohabitating & $384(69.2 \%)$ & $398(70.6 \%)$ & $94(44.5 \%)$ & 77 (45.8\%) \\
\hline \multicolumn{5}{|l|}{ Relationship with child } \\
\hline Biological mother & $341(61.4 \%)$ & $338(59.9 \%)$ & $152(72.0 \%)$ & $119(70.8 \%)$ \\
\hline Biological father & $179(32.3 \%)$ & $183(32.4 \%)$ & $44(20.9 \%)$ & $27(16.1 \%)$ \\
\hline Adoptive mother & $2(0.4 \%)$ & $1(0.2 \%)$ & $0(0.0 \%)$ & $0(0.0 \%)$ \\
\hline Stepfather & $1(0.2 \%)$ & $4(0.7 \%)$ & $0(0.0 \%)$ & $5(3.0 \%)$ \\
\hline Stepmother & $1(0.2 \%)$ & $0(0.0 \%)$ & $0(0.0 \%)$ & $0(0.0 \%)$ \\
\hline Aunt/uncle & $3(0.5 \%)$ & $2(0.4 \%)$ & $0(0.0 \%)$ & $0(0.0 \%)$ \\
\hline Grandparents & $28(5.0 \%)$ & $36(6.4 \%)$ & $15(7.1 \%)$ & $17(10.1 \%)$ \\
\hline \multicolumn{5}{|l|}{ Educational Attainment } \\
\hline No school/Don't know & $112(20.2 \%)$ & $132(23.4 \%)$ & $60(28.4 \%)$ & $38(22.6 \%)$ \\
\hline$<6$ years & $275(49.5 \%)$ & $252(44.7 \%)$ & $97(46.0 \%)$ & $88(52.4 \%)$ \\
\hline$\geq 6$ yrs. Primary & $88(15.9 \%)$ & $89(15.8 \%)$ & $26(12.3 \%)$ & $21(12.5 \%)$ \\
\hline Secondary/vocational school & $80(14.4 \%)$ & $91(16.1 \%)$ & $28(13.3 \%)$ & $21(12.5 \%)$ \\
\hline \multicolumn{5}{|l|}{ Health and safety } \\
\hline Screens positive, disability & $60(10.8 \%)$ & $63(11.2 \%)$ & $36(17.1 \%)$ & $26(15.5 \%)$ \\
\hline Screens positive, depression or anxiety & 275 (49.5\%) & $248(44.0 \%)$ & $117(55.5 \%)$ & $83(49.4 \%)$ \\
\hline Maternal victimisation violence, last three months ${ }^{\mathrm{a}}$ & $78(39.8 \%)$ & $73(35.3 \%)$ & $15(29.4 \%)$ & $15(36.6 \%)$ \\
\hline Paternal perpetration violence, last 3 months ${ }^{a}$ & $38(21.2 \%)$ & $41(22.3 \%)$ & $10(23.3 \%)$ & $4(12.5 \%)$ \\
\hline
\end{tabular}

a among mothers $(n=495)$ and fathers $(n=438)$ who are married or cohabitating HAZ Height-for-age, WHZ weight-for-height, WAZ Weight-for-age 
Table 4 Model-based estimates and effect sizes for primary child development outcomes

\begin{tabular}{|c|c|c|}
\hline \multirow[t]{2}{*}{ Outcomes } & \multicolumn{2}{|c|}{ Mixed model difference in difference estimates ${ }^{\mathbf{a}}$} \\
\hline & Estimated coefficient $(95 \% \mathrm{Cl})$ & $\begin{array}{l}\text { Effect Size } \\
\text { Cohen's d }(95 \% \mathrm{Cl})^{\mathbf{b}} \text { or } \\
\text { Binary: OR }(95 \% \mathrm{Cl})\end{array}$ \\
\hline \multicolumn{3}{|l|}{ CHILD DEVELOPMENT $(N=1084)$} \\
\hline \multicolumn{3}{|l|}{ ECD stimulation in the home } \\
\hline HOME [0-43] (continuous) & $3.85(3.20,4.50)$ & $d=0.87(0.74,0.99)$ \\
\hline OMCI [0-57] (continuous) & $3.06(1.57,4.56)$ & $d=0.29(0.17,0.41)$ \\
\hline FCl (ECD activities) (continuous) & $1.25(1.01,1.48)$ & $d=0.73(0.60,0.86)$ \\
\hline \multicolumn{3}{|l|}{ Child nutrition, health and safety } \\
\hline Dietary Diversity [0-7 food groups] (continuous) & $0.45(0.26,0.64)$ & $d=0.35(0.22,0.47)$ \\
\hline Diarrhoea prevalence (\%) & $-0.28(-0.67,0.11)$ & $0.76(0.51,1.11)$ \\
\hline Diarrhoea care seeking $(\%)^{c}$ & $1.49(0.66,2.31)$ & $4.43(1.95,10.10)$ \\
\hline Fever and cough prevalence (\%) & $-0.18(-0.56,-0.19)$ & $0.83(0.57,1.21)$ \\
\hline Fever and cough care seeking (\%) ${ }^{d}$ & $1.19(0.60,1.77)$ & $3.28(1.82,5.89)$ \\
\hline \multicolumn{3}{|l|}{ Child caretaking practices and child safety } \\
\hline Use of any harsh discipline (\%) & $-1.22(-1.67,-0.76)$ & $0.30(0.19,0.47)$ \\
\hline Exclusive nonviolent discipline (\%) & $0.92(0.16,1.68)$ & $2.50(1.17,5.34)$ \\
\hline \multicolumn{3}{|l|}{ CAREGIVER OUTCOMES $(N=1498)$} \\
\hline \multicolumn{3}{|l|}{ Caregiver mental health } \\
\hline Screens for internalising problems (\%) ${ }^{\mathrm{e}}$ & $-0.54(-0.96,-0.13)$ & $0.58(0.38,0.88)$ \\
\hline \multicolumn{3}{|l|}{ Shared decision-making ${ }^{f}$} \\
\hline Action when child sick (\%) & $0.72(0.27,1.18)$ & $2.06(1.31,3.26)$ \\
\hline What child eats (\%) & $0.35(-0.18,-0.88)$ & $1.43(0.84,2.43)$ \\
\hline \multicolumn{3}{|l|}{ Intimate partner violence } \\
\hline Perpetration, male caregivers (\%) ${ }^{9}$ & $-0.11(-0.97,0.75)$ & $0.90(0.38,2.12)$ \\
\hline Victimisation, female caregivers (\%) ${ }^{\mathrm{h}}$ & $-0.72(-1.43,-0.01)$ & $0.49(0.24,1.00)$ \\
\hline \multicolumn{3}{|l|}{ HOUSEHOLD OUTCOMES $(N=1049)$} \\
\hline \multicolumn{3}{|l|}{ Water, hygiene and sanitation } \\
\hline Place with soap to wash hands (\%) & $0.86(0.42,1.31)$ & $2.37(1.52,3.69)$ \\
\hline Water treatment (\%) & $1.22(0.77,1.67)$ & $3.39(2.16,5.30)$ \\
\hline Accessing clean water (\%) & $0.65(0.01,1.29)$ & $1.91(1.01,3.62)$ \\
\hline
\end{tabular}

HOME Home Observation for Measurement of the Environment, OMCI The Observation of Mother-Child Interaction, $\mathrm{FCl}$ Family Care Indicators

${ }^{a}$ Coefficients and effect sizes represent the "difference-in-difference" or "time-by-treatment" interaction between the two groups

b Cohen's $d$ estimated from the regression coefficient for continuous outcomes using the pooled standard deviation of the outcome at baseline

c Among those with prevalent diarrhoea ( $N=376$ at baseline and $N=394$ at post-intervention)

${ }^{d}$ Among those with prevalent fever or cough ( $N=595$ at baseline and $N=707$ at post-intervention)

e Scored $\geq 1.75$ on the Hopkins Symptom Checklist-25 Questionnaire

${ }^{f}$ Among married or cohabitating mothers and fathers $(N=913)$

${ }_{9}^{9}$ Among male caregivers reporting a current intimate partner at baseline $(N=450)$

${ }^{\mathrm{h}}$ Among female caregivers reporting a current intimate partner at baseline $(N=523)$

continuous outcomes and frequencies for binary outcomes can be found in Additional File 4.

\section{Positive parenting and responsive care}

Compared with UC, children receiving Sugira Muryango experienced improvements in caregiver engagement scored on the HOME, the OMCI, and the FCI. Improvements on the HOME inventory were 3.9 points greater among intervention families compared with UC
(Coefficient $=3.85,95 \%$ CI: 3.20, 4.50; Cohen's $d=0.87$, 95\% CI: 0.74, 0.99). Improvements on the OMCI total score were 3.1 points greater among intervention families than UC (Coefficient $=3.06,95 \%$ CI: 1.57, 4.56; Cohen's $d=0.29,95 \%$ CI: $0.17,0.41)$. The increase in stimulating caregiving activities (FCI) was 1.2 activities greater in intervention families compared with UC (Coefficient $=1.25,95 \%$ CI: 1.01, 1.48; Cohen's $d=0.73,95 \%$ CI $0.60,0.86)$. 
Dietary diversity, child health, care seeking, and hygiene Pre- to post-intervention, families receiving Sugira Muryango reported a significant increase of 0.45 food groups in children's dietary diversity compared to UC (Coefficient $=0.45$, 95\% CI: 0.26; 0.64, Cohen's $d=0.35$, CI: $0.22,0.47)$. Pre- to post-intervention the prevalence of acute childhood illnesses was unchanged; however, at post-intervention, the improvement in odds of seeking care for child diarrhoea were 4.4 times the higher in families receiving Sugira Muryango relative to UC (Coefficient $=1.49,95 \%$ CI: $0.66,2.31 ; \quad \mathrm{OR}=4.43,95 \%$ CI: $1.95,10.10)$ and 3.3 times higher in seeking care for child fever in families receiving Sugira Muryango relative to UC (Coefficient $=1.19,95 \% \mathrm{CI}: 0.60,1.77$; OR $=3.28$, 95\% CI: 1.82, 5.89).

\section{Violence and harsh discipline}

Following the 12-module intervention, the odds of exposure to harsh discipline decreased $70 \%$ more in families receiving Sugira Muryango compared to UC children (Coefficient $=-1.22$, 95\% CI: $-1.67,-0.76$; $\mathrm{OR}=0.30,95 \% \mathrm{CI}: 0.19,0.47$ ). Moreover, the odds of being exclusively exposed to nonviolent forms of discipline increased 2.5 more for children in Sugira Muryango families compared to UC (Coefficient $=0.92,95 \%$ CI: 0.16, 1.68; OR $=2.50,95 \%$ CI:1.17, 5.34). Sugira Muryango was also associated with a $51 \%$ decrease in the odds of females reporting victimisation to intimate partner violence $($ Coefficient $=-0.72,95 \% \mathrm{CI}:-1.43,-0.01)$; $\mathrm{OR}=0.49,95 \% \mathrm{CI}: 0.24,1.00)$. We did not observe intervention related differences in the changes in father reports of intimate partner violence perpetration.

\section{Parental mental health and shared decision-making}

The intervention was associated with significant improvement in anxiety and depression symptoms among caregivers receiving Sugira Muryango compared to UC (Coefficient $=-0.54, \mathrm{CI}:-0.96,-0.13$; OR $=0.58,95 \%$ CI:0.38, 0.88). Dual-caregiver dyads receiving Sugira Muryango did not show increased shared decisionmaking regarding child feeding, but had twice the increase in odds of jointly deciding what to do when the child was sick (Coefficient $=0.72,95 \%$ CI: 0.27, 1.18; $\mathrm{OR}=2.06,95 \%$ CI: $1.31,3.26$ ).

\section{Water, hygiene and sanitation}

Sugira Muryango households also had 2.4 times greater improvement in odds of engaging in handwashing with soap (Coefficient $=0.86,95 \%$ CI $0.42,1.31$; OR $=2.37$, 95\% CI: 1.52, 3.69) and 3.4 times greater improvement in odds of engaging in safe water treatment (Coefficient $=1.22$, 95\% CI: $0.77,1.67 ; \mathrm{OR}=3.39,95 \%$ CI: 2.16 , 5.30) following the intervention compared with UC. Intervention households also had almost twice the odds in improvement in access to clean water in the intervention group relative to UC (Coefficient $=0.65,95 \% \mathrm{CI}$ : 0.01, 1.29; OR = 1.91, 95\% CI: 1.01, 3.62).

\section{Discussion}

Sugira Muryango was designed to address the needs of Rwanda's most vulnerable families. Through home visiting, we involved a range of family members, including fathers, in nurturing care. We observed that active coaching, play, alternatives to harsh discipline and violence, and encouragement of family strengths can help vulnerable households create a better home and care environment for young children drawing on formal and nonformal resources. Results indicate that this brief (1216 week) intervention led to improvements in caregiving practices related to child development including parentchild interactions and stimulation, nutrition, care seeking, and reduced violence. The observed effect sizes for changes in ECD-related parent behaviours fall within the range found in other home-visiting ECD interventions in LMICs. For example, our effect size for the HOME $(d=0.87)$ is comparable to effect sizes reported in previous studies in Pakistan [4] $(d=0.30)$, Uganda [19] $(d=1.1)$ and Bangladesh [5] ( $d=0.55$ to $d=0.68)$, and our effect size for the OMCI $(d=0.29)$ is comparable to that reported in Pakistan ( $d=0.20$ to 0.80$)$ [4]. The observed effect size for dietary diversity $(d=0.35)$ is also comparable to effect sizes $(d=0.54)$ in a parenting intervention in Uganda [19] and in Bangladesh $(d=0.40)$ [5]. Although we did not see improvement in the prevalence of acute childhood illness in the intervention group, we did see improvements related to care seeking if children were sick. The absence of improvements in children's health status was somewhat surprising given the impact of the intervention on caregiver behaviours that are known to improve child health including dietary diversity and improved hygiene. Two factors may explain the lack of improvement in child health. First, given the four-month interval between the baseline and postintervention assessment, the season changed from rainy season (April/May) to dry season (August/September), which may impact the prevalence of childhood disease. A second explanation may be that children age over the course of the intervention and assessments and may become less prone to illness both in the intervention and the UC group as they age. Sugira Muryango was also associated with reductions in family violence-reduced use of harsh punishment practices and victimisation of mothers by intimate partner violence. Moreover, increased shared decision-making about what to do if a child was sick was indicative of increased father involvement in childcare.

Limitations must be noted. First, in this brief assessment period, we did not explore physical and cognitive 
development outcomes; these will be examined at 12month follow up. Second, some measures relied on parent-report and could suffer from differential bias because parents who were exposed to the intervention may have been more aware of socially desirable responses. We also see a discrepancy between females' reports of victimisation and males' reports of perpetration of violence suggesting that male caregivers may not give accurate self-reports. Relatedly, we note that we limited this examination of intimate partner violence to victimisation of mothers and perpetration by fathers although we recognise other forms of intimate partner violence exist. On the other hand, a strength of the study is that key outcomes related to nurturing care, such as the OMCI and the HOME were reported by a blinded, trained observer. Third, Sugira Muryango was delivered to vulnerable households categorised as extremely poor, as defined by eligibility to a social protection programme offered to Ubudehe 1 households in Rwanda. The extreme level of poverty in these households may limit the generalisability of the results to other, less poor households. Future studies may examine how families of different socioeconomic status may benefit from the programme.

\section{Conclusion}

Family home-visiting interventions like Sugira Muryango have an important role to play in promoting ECD and preventing family violence globally. The integration of ECD programmes and social protection agendas is a promising area for helping vulnerable children and families break intergenerational cycles of poverty and violence.

\section{Supplementary information}

Supplementary information accompanies this paper at https://doi.org/10. 1186/s12889-020-08693-7.

\section{Additional File 1. Adverse Events \\ Additional File 2. Intra-class correlational \\ Additional File $\mathbf{3}$ Interaction by VUP status. Difference in the effects of the intervention emerged between CPW and ePW households on the HOME inventory $(p=0.002)$, shared decision making about what to do when a child is sick ( $p=0.016)$, shared decision making about what a child eats $(p=0.020)$, and perpetration of violence $(p=0.031)$. A full table of results is in the supplemental material, Table S3. On the HOME inventory, we found that the intervention effect was greater in the ePW households compared with CPW households. For the shared decision- making questions, we found that intervention effects were limited to CPW households. For perpetration of violence we found that intervention effects were limited to ePW households.}

Additional File 4. Baseline and post-intervention means. Unadjusted raw means from unimputed dataset.

\section{Abbreviations}

CBC: Community-based coach; DHS: Demographic and Health Surveys; CPW: Classic public works programme; ECD: Early child development; ePW: Expanded public works programme; FCl: Family Care Indicators: HOME: Home Observation for Measurement of the Environment; LMICs: Low- and middle-income countries; MICS: Multiple Indicator Cluster Survey; OMCl: Observation of Mother Child Interaction; UC: Usual Care;

UNICEF: United Nations International Children's Emergency Fund; VUP: Vision 2020 Umurenge Programme; WHO: World Health Organization

\section{Acknowledgments}

The work was made possible by the collaboration of the University of Rwanda Center for Mental Health, Ministry of Gender and Family Promotion and the National Early Childhood Development Program under the direction of National Coordinator Dr. Anita Aismwee and the advisory committee comprised of representatives from the Ministry of Local Government, National Children's Commission, Rwanda Biomedical Centre, and the University of Rwanda School of Nursing. We would like to thank Matias Placencio-Castro and Dr. Simo Goshev for their help with data analysis.

\section{Authors' contributions}

TSB conceptualised the study, obtained funding, led intervention development of the intervention, and provided supervision. SKJ and DB conducted data analyses and contributed to data interpretation and writing. RTB contributed to study concept and design, data analysis, interpretation of the data, and drafting of the manuscript. SM oversaw intervention implementation and data collection and made significant intellectual contributions to the manuscript content. JF oversaw the fidelity monitoring process and provided input during intervention development. KG, OU, and $\mathrm{SB}$ helped refine the curriculum and were engaged in direct supervision of the CBCs and families. AK, LR, and BW contributed to policy dialogue management with government counterparts, study design, significantly securing funding and writing and data interpretation. VS supported clinical supervision during intervention delivery, contributed to intervention adaptation and made significant intellectual contributions to the intervention and manuscript content. AY assisted in further curriculum refinements and contributed to the manuscript writing. All authors have read and approved the manuscript

\section{Funding}

This study was funded by The World Bank Early Learning Partnership (Grant Number 7170035), the Strategic Impact Evaluation Fund and the Japan Trust (Grant Number 7186617), USAID Rwanda (Grant Number AID-696-A-1600003), the Network of European Foundations (CVECF-BOSTON COLLEGE_2017), and ELMA Philanthropies (Grant number 16-F0018-BC). Coauthors from the World Bank were involved in the study design, interpretation of data, and writing of the manuscript. None of the other funders were involved in study design, data collection, data analysis, data interpretation, or writing. This work does not necessarily reflect the views of the World Bank, its Board of Executive Directors, or the governments they represent. The World Bank does not guarantee the accuracy of the information included in this work. Dr. Betancourt maintained authority over all decision-making over design, analysis, interpretation, and publication. All authors have indicated they have no financial relationships relevant to this article to disclose and have no conflicts of interest relevant to this article to disclose.

\section{Availability of data and materials}

Deidentified individual participant data will not be made available since this is an ongoing trial. De-identified data will be available from the authors 6 months after collection of the 12-month follow-up. The dataset supporting the conclusions of this article are included within the article (and its additional files)

Ethics approval and consent to participate

Ethical approval was obtained from the institutional review boards of the Harvard T. H. Chan School of Public Health and Boston College as well as the Rwanda National Ethics Committee, National Committee for Science and Technology, and the National Institute of Statistics of Rwanda. De-identified data will be available from the authors 6 months after collection of the 12month follow-up. All adult study participants provided written informed consent for their own participation and primary caregivers gave written consent for participation of their children. 


\section{Competing interests}

The authors declare that they have no competing interest.

\section{Author details}

'Boston College School of Social Work, McGuinn Hall 106M, 140 Commonwealth Avenue, Chestnut Hill, MA 02467, USA. ${ }^{2}$ Department of Epidemiology, Harvard School of Public Health, Boston, MA, USA. ${ }^{3}$ Women's Study Research Center, Brandeis University, Waltham, MA, USA. ${ }^{4}$ Department of Global Health and Population, Harvard School of Public Health, Boston, MA, USA. ${ }^{5}$ FXB Rwanda, Kigali, Rwanda. ${ }^{6}$ The World Bank, Washington, DC, USA. ${ }^{7}$ College of Medicine and Health Sciences, University of Rwanda, Kigali, Rwanda.

Received: 10 August 2019 Accepted: 13 April 2020

Published online: 06 May 2020

\section{References}

1. Black MM, Walker SP, Fernald LCH, et al. Early childhood development coming of age: science through the life course. Lancet. 2017;389:77-90.

2. Andersen SL. Trajectories of brain development: point of vulnerability or window of opportunity? Neurosci Biobehav Rev. 2003;27:3-18.

3. Britto PR, Lye SJ, Proulx K, et al. Nurturing care: promoting early childhood development. Lancet. 2017;389:91-102.

4. Yousafzai AK, Rasheed MA, Rizvi A, Armstrong R, Bhutta ZA. Effect of integrated responsive stimulation and nutrition interventions in the lady health worker programme in Pakistan on child development, growth, and health outcomes: a cluster-randomised factorial effectiveness trial. Lancet. 2014;384:1282-93.

5. Aboud FE, Singla DR, Nahil MI, Borisova I. Effectiveness of a parenting program in Bangladesh to address early childhood health, growth and development. Soc Sci Med. 2013;97:250-8.

6. Richter LM, Daelmans B, Lombardi J, et al. Investing in the foundation of sustainable development: pathways to scale up for early childhood development. Lancet. 2017;389:103-18.

7. Mikton C, MacMillan H, Dua T, Betancourt TS. Integration of prevention of violence against children and early child development. Lancet Glob Heal. 2014;2:e442-3.

8. National Institute of Statistics of Rwanda. The fifth integrated household living survey (EICV5) Rwanda poverty profile report, 2016/17. 2018 http:// www.statistics.gov.rw/publication/eicv-5-rwanda-poverty-profile-report201617.

9. The World Bank. Poverty. 2019. https://www.worldbank.org/en/topic/ poverty/overview. Accessed 20 Dec 2019.

10. Republic of Rwanda Local Administrative Entities Development Agency (LODA). Environmental and social management framework for the social protection investment project financing operation. 2018. https://loda.gov. rw/fileadmin/user_upload/documents/2014_PRO/Documents/Revised_SP_ IPF_ESMF for_Disclosure March_2018.pdf. Accessed 1 Mar 2020.

11. Government of Rwanda. Economic Development and Poverty Reduction Strategy 2013-2018: Shaping our Development 2013. https://extranet.who. int/nutrition/gina/en/node/22998.

12. Ministry of Health Rwanda, UNICEF Rwanda. Knowledge, Attitudes and Practices Assessment on Early Nurturing of Children Report. 2014.

13. Republic of Rwanda Local Administrative Entities Development Agency (LODA). Ubudehe social categorization report. 2016.

14. Betancourt TS, Ng LC, Kirk CM, et al. Family-based promotion of mental health in children affected by HIV: a pilot randomized controlled trial. J Child Psychol Psychiatry. 2017;58:922-30.

15. Betancourt TS, Franchett E, Kirk CM, et al. Integrating social protection and early childhood development: open trial of a family home-visiting intervention, Sugira Muryango. Early Child Dev Care. 2018. https://doi.org/10. 1080/03004430.2018.1464002

16. World Health Organization, UNICEF. Care for child development: improving the care for young children. 2012 https://www.who.int/maternal_child_ adolescent/documents/care child_development/en/.

17. Rasheed MA, Yousafzai AK. The development and reliability of an observational tool for assessing mother-child interactions in field studiesexperience from Pakistan. Child Care Health Dev. 2015;41:1161-71.

18. Caldwell BM, Bradley RH. Home observation for measurement of the environment: administration manual; 2003.
19. Singla DR, Kumbakumba E, Aboud FE. Effects of a parenting intervention to address maternal psychological wellbeing and child development and growth in rural Uganda: a community-based, cluster-randomised trial. Lancet Glob Heal. 2015;3:e458-69.

20. UNICEF. MICS5 questionnaire for children under five. 2013. http://mics. unicef.org/tools?round=mics5.

21. USAID, AED, Food and nutrition technical assistance, UC Davis, International Food Policy Research Institute, WHO. Indicators for assessing infant and young child feeding practices. Geneva; 2008. http://whqlibdoc.who.int/ publications/2008/9789241596664_eng.pdf. Accessed 1 Mar 2020.

22. National Institute of Statistics of Rwanda, Ministry of Finance and Economic Planning, Ministry of Health, ICF International. Rwanda Demographic and Health Survey 2014-15. Kigali; 2016. https://dhsprogram.com/pubs/pdf/ FR316/FR316.pdf. Accessed 1 Mar 2020.

23. National Institute of Statistics of Rwanda, ORC Macro. Rwanda Demographic and Health Survey 2005. Kigali; 2006. https://dhsprogram.com/pubs/pdf/FR1 83/FR183.pdf. Accessed 1 Mar 2020.

24. UNICEF, Imbuto Foundation. Early Childhood Development and Family Services: baseline evaluation in 20 sites in Rwanda. 2015 https://www.unicef. org/evaldatabase/files/ECD_and_F_Baseline_Evaluation_Rwanda.pdf.

25. Bolton P. Local perceptions of the mental health effects of the Rwandan genocide. J Nerv Ment Dis. 2001;189:243-8.

26. Plumpton CO, Morris T, Hughes DA, White IR. Multiple imputation of multiple multi-item scales when a full imputation model is infeasible. BMC Res Notes. 2016;9:45

27. World Health Organization, UNICEF. WHO child growth standards and the identification of severe acute malnutrition in infants and children. 2009.

\section{Publisher's Note}

Springer Nature remains neutral with regard to jurisdictional claims in published maps and institutional affiliations.
Ready to submit your research? Choose BMC and benefit from:

- fast, convenient online submission

- thorough peer review by experienced researchers in your field

- rapid publication on acceptance

- support for research data, including large and complex data types

- gold Open Access which fosters wider collaboration and increased citations

- maximum visibility for your research: over $100 \mathrm{M}$ website views per year

At BMC, research is always in progress.

Learn more biomedcentral.com/submissions 